$\underline{\text { Preprint typeset in JHEP style - HYPER VERSION }}$

hep-th/0209209

TUW-02-09

\title{
Graded Poisson-Sigma Models and Dilaton-Deformed 2D Supergravity Algebra
}

\author{
Luzi Bergamin and Wolfgang Kummer \\ Institute for Theoretical Physics, Technical University of Vienna, Wiedner Hauptstr. \\ 8-10, 1040 Vienna, Austria \\ E-mail: bergamin@tph.tuwien.ac.at, wkummer@tph.tuwien.ac.at
}

\begin{abstract}
Fermionic extensions of generic 2d gravity theories obtained from the graded Poisson-Sigma model (gPSM) approach show a large degree of ambiguity. In addition, obstructions may reduce the allowed range of fields as given by the bosonic theory, or even prohibit any extension in certain cases. In our present work we relate the finite $\mathrm{W}$-algebras inherent in the gPSM algebra of constraints to algebras which can be interpreted as supergravities in the usual sense (Neuveu-Schwarz or Ramond algebras resp.), deformed by the presence of the dilaton field. With very straightforward and natural assumptions on them -like demanding rigid supersymmetry in a certain flat limit, or linking the anti-commutator of certain fermionic charges to the Hamiltonian constraint- in the "genuine" supergravity obtained in this way the ambiguities disappear, as well as the obstructions referred to above. Thus all especially interesting bosonic models (spherically reduced gravity, the Jackiw-Teitelboim model etc.) under these conditions possess a unique fermionic extension and are free from new singularities. The superspace supergravity model of Howe is found as a special case of this supergravity action. For this class of models the relation between bosonic potential and prepotential does not introduce obstructions as well.
\end{abstract}

KeYwords: Dilaton supergravity, graded Poisson-Sigma model, superconformal algebra. 


\section{Contents}

1. Introduction

2. Covariant algebra in (graded) PSM 6

2.1 (g)PSM Hamiltonian constraints 6

2.2 Conformal algebra for PSMs

3. Algebras for generic gPSM gravity 8

3.1 Rigid supersymmetry 9

3.2 Algebra for generic gPSMs 10

4. Dilaton prepotential algebra: a special example 13

5. General deformed superconformal algebra 16

6. General deformed supergravity 20

7. Conclusions 21

A. Notations and conventions 22

\section{Introduction}

Diffeomorphism invariant dilaton theories in $1+1$ dimensions for some time have been a promising field in classical and quantum theory. They include effective theories of direct physical interest, like reduced d-dimensional Einstein theories and the extensions thereof (Einstein-deSitter, Jordan-Brans-Dicke theories [1-5]), but also theories suggested by stringy arguments $[6]^{1}$. On the other hand, supersymmetric extensions of gravity [8-11] are believed to be a crucial ingredient for a consistent solution of the problem how to quantize gravity in the framework of string/brane theory [12-14].

Much of the recent progress $[7,15-18]$ to understand bosonic gravity theories in two dimensions also at the quantum level is based upon the equivalence $[19,20]$ of a torsion free general dilaton theory [21-25] and a Hamiltonian action of the type of a Poisson-Sigma model (PSM) $[26,27]$. A (graded) PSM ((g)PSM) is defined by the action

$$
\begin{aligned}
\mathcal{S} & =\int_{M} \mathrm{~d} X^{I} \wedge A_{I}+\frac{1}{2} P^{I J} A_{J} \wedge A_{I} \\
& =\int \mathrm{d}^{2} x\left(\partial_{0} X^{I} A_{1 I}-\partial_{1} X^{I} A_{0 I}+P^{I J} A_{0 J} A_{1 I}\right) .
\end{aligned}
$$

\footnotetext{
${ }^{1} \mathrm{~A}$ recent review with rather extensive literature is represented by ref. [7].
} 
Target space coordinates $X^{I}(x)$ and one-form gauge-fields $A_{I}=A_{m I}(x) \mathrm{d} x^{m}$ live upon the $2 \mathrm{~d}$ manifold $M$. The dynamics is encoded in the Poisson tensor $P^{I J}(X)$, which must have a vanishing (graded) Nijenhuis tensor ${ }^{2}$ (obey the Jacobi identity for a (graded) Schouten bracket defined as $\left[X^{I}, X^{J}\right]=P^{I J}$ )

$$
P^{I L} \partial_{L} P^{J K}+g-p e r m(I J K)=0 \text {. }
$$

Then the action (1.1) is invariant under the symmetry transformations

$$
\delta X^{I}=P^{I J} \epsilon_{J}, \quad \delta A_{I}=-\mathrm{d} \epsilon_{I}-\left(\partial_{I} P^{J K}\right) \epsilon_{K} A_{J},
$$

where the term $\mathrm{d} \epsilon_{I}$ in the second of these equations provides the justification for calling $A_{I}$ "gauge fields".

For a generic (g)PSM the commutator of two transformations (1.3) is a symmetry modulo the equations of motion. Only for $P^{I J}$ linear in $X^{I}$ a closed (and linear) Lie algebra is obtained and (1.2) reduces to the Jacobi identity for the structure constants of a Lie group. If the Poisson tensor is singular -the actual situation in any application to $2 \mathrm{~d}$ (super-)gravity due to the odd dimension of the bosonic part of the tensor- there exist (one or more) Casimir functions $C(X)$ obeying

$$
\left[X^{I}, C\right]=P^{I J} \frac{\partial C}{\partial X^{J}}=0
$$

which, when determined by the field equations of motion, are constants of motion.

So far the number of target space coordinates (the range of the indices $I, J$ etc.) has not been specified. In the following we identify ${ }^{3}$ the first component $X^{0}$ of $X^{I}$ with the dilaton field $\phi$ and use the symbol $\phi$ also to describe that value of $I: X^{0}=X^{\phi}=\phi$. The corresponding gauge field becomes $A_{0}=A_{\phi}=\omega$. In the application to bosonic gravity ${ }^{4}$ $A_{I}$ comprises the Cartan variables spin connection $\omega_{a b}=\omega \epsilon_{a b}$ and zweibeine $e_{a}$. The corresponding three target space coordinates are $X^{I}=X^{i}=\left(\phi, X^{a}\right)$. The component $P^{\phi a}=X^{b} \epsilon_{b}^{a}$ is determined by local Lorentz invariance. Only this choice leads to the correct covariant derivative $(D e)_{a}=\mathrm{d} e_{a}-\omega \epsilon_{a}{ }^{b} e_{b}$ in the 2 d gravity action (cf. eq. (A.8)). In the pure bosonic case $P^{i j}=-P^{j i}$ is anti-symmetric. Therefore, the only remaining components of the Poisson tensor can be written as $P^{a b}=v \epsilon^{a b}$, where the locally Lorentz invariant "potential" $v=v(\phi, Y)$ describes different models $\left(Y=X^{a} X_{a} / 2\right)$. Evaluating (1.1) with that $P^{\phi a}$ and $P^{a b}$ the action $\left(\epsilon=\frac{1}{2} \epsilon^{a b} e_{b} \wedge e_{a}\right)$

$$
\mathcal{S}_{\text {bosonic }}=\int_{\mathcal{M}}\left(\phi \mathrm{d} \omega+X^{a} D e_{a}+\epsilon v\right)
$$

\footnotetext{
${ }^{2}$ Here and in the following $\partial_{K}=\frac{\partial}{\partial X^{K}}$ represents the derivative with respect to the target space variables. More details of the conventions are given in the Appendix.

${ }^{3}$ Further explanations of the usage of indices are given in the Appendix.

${ }^{4}$ The condition to identify the PSM 1-forms directly with the geometrical variables can be relaxed, leading to more general gravity theories. Given the mathematical difficulties to find explicit solutions with vanishing Nijenhuis tensor even for the present situation, we restrict the discussion to this direct identification.
} 
is obtained. The most interesting models are described by potentials of type

$$
v=Y Z(\phi)+V(\phi),
$$

where e.g. spherically reduced gravity from d dimensions is given by [28-31]

$$
Z_{S R G}=-\frac{d-3}{(d-2) \phi}, \quad V_{S R G}=-\lambda^{2} \phi^{(d-4) /(d-2)},
$$

with the CGHS model [6] as the formal limit $d \rightarrow \infty$. The Jackiw-Teitelboim model [32-36] corresponds to

$$
Z_{J T}=0, \quad V_{J T}=-\Lambda \phi,
$$

and the bosonic part of the simplest non-trivial $2 \mathrm{~d}$ supergravity model of Howe [37] -after the correct identification of the dilaton with one of the components of the superfield [38]becomes

$$
Z_{H}=0, \quad V_{H}=-\frac{1}{2} \phi^{2} .
$$

Potentials of type (1.6) allow the integration of the (single) Casimir function $C$ in (1.4)

$$
C=e^{Q(\phi)} Y+W(\phi) \quad Q(\phi)=\int_{\phi_{1}}^{\phi} \mathrm{d} \varphi Z(\varphi) \quad W(\phi)=\int_{\phi_{0}}^{\phi} \mathrm{d} \varphi e^{Q(\varphi)} V(\varphi)
$$

where e.g., in spherically reduced gravity (1.7) $C$ on-shell simply is proportional to the ADM-mass in the Schwarzschild solution.

The auxiliary variables $X^{a}$ and the torsion-dependent part of the spin connection can be eliminated by algebraic equations of motion. Then the action reduces to the familiar generalized dilaton theory in terms of the dilaton field $\phi$ and the metric:

$$
\mathcal{S}_{G D T}=\int \mathrm{d}^{2} x e\left(\frac{1}{2} R \phi-\frac{1}{2} Z \partial^{m} \phi \partial_{m} \phi+V\right)
$$

Both formulations are equivalent at the classical $[19,20]$ as well as at the quantum level [16-18]. But the PSM formulation possesses crucial technical advantages, another of which is the topic of our present work.

A generalization of $\mathcal{S}_{G D T}$ to supergravity exists for a long time [37,39]. However, in order to exploit the advantages of the formulation as a "Hamiltonian" one in (1.5), a supergravity extension of that action is desirable. All gravity models with $Y$-dependent potentials in the PSM formulation $(Z \neq 0$ in (1.6) $)$ possess non-vanishing bosonic torsion which turned out to be an extremely cumbersome feature in all attempts to formulate directly corresponding $2 \mathrm{~d}$ supergravities in the superfield formalism. The conventional constraints for vanishing bosonic torsion are well studied. Now they have to be changed in a highly nontrivial manner. As shown in [40] the entire procedure of solving Bianchi identities for superfields with a large number of nontrivial components must be started from scratch, if the bosonic torsion does not vanish any more. In contrast, the PSM extends straightforwardly by the introduction of $N$ additional fermionic target space Majorana 
coordinates $\chi^{(i) \alpha}$ ("dilatinos") and corresponding "gravitinos" $\psi_{\alpha}^{(i)}(i=1, \ldots N)$ in $X^{I}$, resp. $A_{I}$

$$
X^{I}=\left(X^{i}, X^{(n) \alpha}\right)=\left(\phi, X^{a}, \chi^{(n) \alpha}\right), \quad A_{I}=\left(A_{i}, A_{\alpha}^{(n)}\right)=\left(\omega, e_{a}, \psi_{\alpha}^{(n)}\right)
$$

to a gPSM with a graded Poisson tensor $P^{I J}=-(-1)^{I J} P^{J I}[38,41]$. Thanks to our conventions (cf. Appendix) for summation of adjacent indices in eqs. (1.1)-(1.4) all formulas remain unchanged. This generalization directly provides an $(N, N)$ "supergravity" theory without auxiliary fields and thus without the need to impose constraints, to solve Bianchi identities etc. As can be seen from the second eq. in (1.3) the solution of the graded counterpart of that equation contains a supergravity-like transformation law for the gravitino-field $\psi_{\alpha}$, deformed by the dilaton fields.

When a bosonic potential, as exemplified by (1.6), is given, the solution of the graded counterpart of the Jacobi identity (1.2) provides all possible fermionic extensions. It turned out that an algebraic general solution ${ }^{5}$ of this problem can be given explicitly [38]. However, this solution was found to be far from unique. As in ref. [38], in the present work we also concentrate on $N=(1,1)$ supergravity, which must be realized as a subset of such gPSM theories with one field $\chi^{\alpha}$ in a non-degenerate fermionic extension (rank 2). In this case [38] the general solution for a certain bosonic potential $v$ depends on no less than five arbitrary Lorentz covariant functions. In addition, the new fermionic terms in the algebras and in the ensuing Lagrangians produced by this method exhibit new singularities (obstructions) in the variables $\phi$ and $Y$ in points where the original bosonic potential was regular. For certain bosonic models any fermionic extension is prohibited. The basic reason for the latter behaviour is that the potential (1.6) in the extended case must be derivable from a prepotential.

Attempts to simply require the absence of new singularities in the fermionic part of the action as a means to determine "allowed" extensions turned out to be unsuccessful: In a counter-example [38] two different supergravities with the same (bosonic) $v$ of spherical reduction (1.6) with (1.7)) were found to be free from such singularities.

Therefore, the question arises how the additional structure present in standard supergravity theories formulated in superspace can be found within gPSM gravity models and whether the unpleasant features of a generic gPSM described above are influenced by the ensuing restrictions.

Obviously the nonlinear gauge symmetry (1.3) does not lend itself to an appropriate starting point for this task, the conditions for supergravity being not inherent in general gPSM theories: Any strategy must -in some sense- contain the restriction known in supergravity models from superspace or from a gauge-theoretic approach that, in the limit of flat space-time of the bosonic geometry, the fermionic sector must reduce to rigid supersymmetry [8-11,42]. In a generic PSM the bosonic potential need not have any flat space-time limit and thus a generalization covering also those geometries is necessary. To implement a condition, which is as close as possible to this basic principle, we propose to start from

\footnotetext{
${ }^{5}$ For degenerate fermionic extensions also one or two additional fermionic Casimirs appear beside the basic one in eq. (1.10).
} 
the closed but nonlinear algebra of Hamiltonian constraints $G^{I}=\left(G^{\phi}, G^{a}, G^{\alpha}\right)$, obtained for bosonic PSM-models in refs. [26,43] and consider their straightforward generalizations to gPSMs. However, instead of trying to impose supergravity-type conditions on that nonlinear algebra, we map it onto a linear Virasoro-like algebra deformed by the presence of the dilaton field. This step again is motivated from the study of purely bosonic PSM gravities: Proper linear combinations of the PSM constraints $G^{i}$ derived from $\mathcal{S}_{\text {bosonic }}$ (1.5) are known to become the $\mathrm{ADM}$ constraints $H_{(0)}$ and $H_{(1)}[44,45]$, which are related to lapse and shift in the ADM formulation [46] of gravity. Together with the Lorentz constraint $G^{\phi}$ they form a linear (Virasoro-type) algebra.

The strategy of our paper is to extend that algebra to its graded version derived from a gPSM. In contrast to the bosonic models, this superalgebra will turn out to be extremely complicated. It is neither linear nor does it represent a drastic simplification compared to the algebra of constraints $G^{I}$. In particular it is not obvious to interpret the general result as a deformed Neuveu-Schwarz or Ramond algebra ${ }^{6}$. It rather reflects in a cumbersome way the large arbitrariness in the gPSM as derived by the fermionic extension from a PSM.

Clearly the study of rigid supersymmetry in flat space in the gPSM framework is a necessary first step. It permits the identification of the proper fermionic constraints $I_{( \pm)}$ from $G^{\alpha}$, to be used beside the bosonic ones $\left(H_{(0)}, H_{(1)}, G^{\phi}\right.$ above). On this basis three restrictions appear to be "natural". We propose for "genuine" deformed supergravities that

(1) rigid supersymmetry appears, when the deformation by the dilaton field is removed in flat space.

(2) the mathematical structure of $H_{(0)}, H_{(1)}$ and of the new fermionic generators $I_{( \pm)}$in terms of the Hamiltonian constraints $G^{I}$ (cf. eqs. (3.7) and (3.8) below) is the same as in rigid supersymmetry. In particular we do not allow additional terms in the general form of the generators, whose prefactors vanish in the limit of rigid supersymmetry.

(3) the (anti-commuting) Poisson brackets of the constraints $I_{(+)}$with itself and $I_{(-)}$ with itself lead back to $H_{(0)}$ and $H_{(1)}$ and, at most, the Lorentz constraint $G^{\phi}=G$.

Actually a more precise mathematical formulation will be given in Section 3.2 (requirement (2)) and Section 5 (requirement (3)) after the structure of the general algebra has been set out in detail.

Condition (1) obviously must be fulfilled for any model involving supergravity. The same holds for the first part of (3), which we, though, at first weaken by allowing the appearance of the Lorentz constraint. As will be seen below, however, the conditions (1) and (2) imply that such a dependence does not occur. Requirement (2) implies that the simplest form of $H_{(0)}, H_{(1)}$ and $I_{( \pm)}$compatible with requirement (1) is used.

The very gratifying consequence of (1) to (3), the central result of our paper, is that these requirements not only lead to a nontrivial result, but that the generic problems of

\footnotetext{
${ }^{6}$ In the following, for simplicity, we shall use the term "superconformal" algebra, in agreement the nomenclature used in string theory.
} 
the most general supergravities from gPSMs $[38,40]$ disappear in the particular class of models selected by those requirements. Moreover that class precisely covers the physically interesting models whose bosonic content is given by (1.6)-(1.9).

The paper is organized as follows: In Section 2 we present the algebra of Hamiltonian constraints for a generic gPSM and review the introduction of the ADM constraints for the pure bosonic case. Then the gPSM-formalism is applied to superalgebras containing rigid supersymmetry (Section 3). The three restrictions, set out above, are tested in Section 1 for the model of ref. [47] without bosonic torsion $(Z=0$ in (1.6)), which covers the model of refs. [32-36] and ref. [37] too (cf. (1.8) and (1.9)).

Section 5 is devoted to the analysis of the consequences in the general case, the final form of the superalgebra and the only allowed class of "genuine" supergravity actions as derived from gPSMs are presented in Section 6. In the Conclusion (Sect. 7) we summarize the results. The Appendix contains a summary of our notations.

\section{Covariant algebra in (graded) PSM}

\section{1 (g)PSM Hamiltonian constraints}

The constraints $G^{I}$ for gPSMs are the result of a Hamiltonian analysis of the action (1.1). Canonical variables ${ }^{7}$ and first class primary constraints are defined from the Lagrangian $L$ in (1.1) $\left(\dot{q}^{I}=\partial_{0} X^{I}\right)$ by

$$
\begin{aligned}
X^{I} & =q^{I}, & \bar{q}^{I} & \approx 0, \\
\frac{\partial L}{\partial \dot{q}^{I}} & =p_{I}=A_{1 I}, & \bar{p}_{I} & =A_{0 I} .
\end{aligned}
$$

From the Hamiltonian density $\left(\partial_{1}=\partial\right)$

$$
H=\dot{q}^{I} p_{I}-L=\partial q^{I} \bar{p}_{I}-P^{I J} \bar{p}_{J} p_{I}
$$

the graded canonical equations

$$
\frac{\partial H}{\partial p_{I}}=(-1)^{I} \dot{q}^{I}, \quad \frac{\partial H}{\partial q^{I}}=-\dot{p}_{I}
$$

are consistent with the graded Poisson ${ }^{8}$ bracket for functionals $A$ and $B$

$$
\begin{gathered}
\left\{A, B^{\prime}\right\}=\int_{x^{\prime \prime}}\left[\left((-1)^{A \cdot I} \frac{\delta A}{\delta q^{\prime \prime I}} \frac{\delta B^{\prime}}{\delta p_{I}^{\prime \prime}}-(-1)^{I(A+1)} \frac{\delta A}{\delta p_{I}^{\prime \prime}} \frac{\delta B^{\prime}}{\delta q^{\prime \prime}}\right)+(q \rightarrow \bar{q}, p \rightarrow \bar{p})\right] \\
=\int_{x^{\prime \prime}}\left[\left(\left(\frac{\delta A}{\delta q^{\prime \prime}} \frac{\delta B^{\prime}}{\delta p_{i}^{\prime \prime}}-\frac{\delta A}{\delta p_{i}^{\prime \prime}} \frac{\delta B^{\prime}}{\delta q^{\prime \prime}}\right)+(-1)^{A}\left(\frac{\delta A}{\delta q^{\prime \prime}} \frac{\delta B^{\prime}}{\delta p_{\alpha}^{\prime \prime}}+\frac{\delta A}{\delta p_{\alpha}^{\prime \prime}} \frac{\delta B^{\prime}}{\delta q^{\prime \prime \alpha}}\right)\right)\right. \\
+(q \rightarrow \bar{q}, p \rightarrow \bar{p})],
\end{gathered}
$$

\footnotetext{
${ }^{7}$ The somewhat unusual association of gauge fields as "momenta" and of target space coordinate fields as "coordinates" is appropriate when natural boundary conditions $\left(\delta X^{I}=0\right.$ at $\left.\partial M\right)$ are assumed. Also quantum ordering problems are eliminated in this way [48].

${ }^{8}$ This is the standard Poisson bracket, not to be confused with the Schouten bracket, associated to the Poisson tensor $P^{I J}$ in eqs. (1.2), (1.3).
} 
where $(q \rightarrow \bar{q}, p \rightarrow \bar{p})$ indicates that the functional derivatives have to be performed for both types of variables, with and without bar. The primes indicate the dependence on primed world-sheet coordinates $x$, resp. $x^{\prime}, x^{\prime \prime}$. The Hamiltonian density (2.3)

$$
H=G^{I} \bar{p}_{I}
$$

is expressed in terms of secondary constraints only:

$$
\left\{\bar{q}^{I}, \int \mathrm{d} x^{1} H\right\}=G^{I}=\partial q^{I}+P^{I J} p_{J}
$$

The complete supersymmetric algebra of the gPSM after a straightforward but tedious calculation with the graded Poisson bracket (2.5) takes the simple form $\left(\partial \delta\left(x^{\prime}-x\right)=\right.$ $\left.\partial_{x^{1}} \delta\left(x^{1}-x^{1}\right)\right)$

$$
\begin{aligned}
\left\{q^{I}, p_{J}^{\prime}\right\} & =(-1)^{I} \delta\left(x-x^{\prime}\right) \delta_{J}^{I}, \\
\left\{\bar{q}^{I}, \bar{p}_{J}^{\prime}\right\} & =(-1)^{I} \delta\left(x-x^{\prime}\right) \delta_{J}^{I}, \\
\left\{q^{I}, \bar{p}_{J}^{\prime}\right\} & =\left\{\bar{q}^{I}, p_{J}^{\prime}\right\}=0 \\
\left\{G^{I}, G^{\prime}\right\} & =-G^{K} \partial_{K} P^{I J} \delta\left(x-x^{\prime}\right), \\
\left\{G^{I}, \bar{p}_{J}^{\prime}\right\} & =\left\{G^{I}, \bar{q}^{J}\right\}=0 \\
\left\{G^{I}, p_{J}^{\prime}\right\} & =(-1)^{I} \partial \delta\left(x-x^{\prime}\right) \delta_{J}^{I}+(-1)^{I J} \partial_{J} P^{I K} p_{K} \delta\left(x-x^{\prime}\right), \\
\left\{G^{I}, q^{\prime J}\right\} & =-P^{I J} \delta\left(x-x^{\prime}\right),
\end{aligned}
$$

which is seen to preserve the structure of the bosonic case [43]. Eq. (2.9) implies that the constraint algebra in general is a finite $W$-algebra with structure functions $f_{K}{ }^{I J}=\partial_{K} P^{I J}$. It simplifies to a Lie-algebra if the Poisson tensor is linear in the target-space coordinates, only.

\subsection{Conformal algebra for PSMs}

It is a remarkable result for non-supersymmetric models that such a $W$-algebra in the absence of fermionic fields can be mapped onto the standard current-algebra of conformal symmetry (Virasoro algebra). In ref. [44] for two dimensional gravity with (the anholonomic index $a$ is expressed in light-cone components, cf. Appendix)

$$
\begin{aligned}
& q^{i}=\left(q^{\phi}, q^{a}\right)=\left(\phi, X^{++}, X^{--}\right), \\
& p_{i}=\left(p_{\phi}, p_{a}\right)=\left(\omega_{1}, e_{1 \mid++}, e_{1 \mid--}\right), \quad \bar{p}_{i}=\left(\bar{p}_{\phi}, \bar{p}_{a}\right)=\left(\omega_{0}, e_{0 \mid++}, e_{0 \mid--}\right),
\end{aligned}
$$

from an ADM analysis [46] a different form of the Hamiltonian density $H=N_{(0)} H_{(0)}+$ $N_{(1)} H_{(1)}+B_{(0)} G$ was obtained. Its constraints become Lorentz invariant linear combinations of the $G^{i}=\left(G^{\phi}, G^{a}\right)=\left(G^{\phi}, G^{++}, G^{--}\right)$:

$$
H_{(0)}=-G^{a} \epsilon_{a}^{b} p_{b}=G^{++} p_{++}-G^{--} p_{--} \quad H_{(1)}=G^{a} p_{a}=G^{++} p_{++}+G^{--} p_{--}
$$


Here $H_{(0)}$ and $H_{(1)}$ are related to the lapse $N_{(0)}$ and shift $N_{(1)}$, respectively. Because of (2.8)-(2.10) they generate another algebra ("deformed ADM algebra") with the Lorentz constraint $G^{\phi}=G$ mixed in:

$$
\begin{aligned}
\left\{H_{(0)}, H_{(0)}^{\prime}\right\} & =\left(H_{(1)}+H_{(1)}^{\prime}\right) \partial \delta\left(x-x^{\prime}\right) \\
\left\{H_{(0)}, H_{(1)}^{\prime}\right\} & =\left(H_{(0)}+H_{(0)}^{\prime}\right) \partial \delta\left(x-x^{\prime}\right)+2 G\left(\partial_{\phi} P^{--k}\right) p_{k} p_{--} \delta\left(x-x^{\prime}\right) \\
\left\{H_{(1)}, H_{(1)}^{\prime}\right\} & =\left(H_{(1)}+H_{(1)}^{\prime}\right) \partial \delta\left(x-x^{\prime}\right) \\
\left\{G, H_{(0)}^{\prime}\right\} & =\left\{G, H_{(1)}^{\prime}\right\}=\left\{G, G^{\prime}\right\}=0
\end{aligned}
$$

By a modification of the ADM constraints [45] in terms of $G=G^{\phi}$ and

$$
\begin{aligned}
\tilde{H}_{(0)} & =G^{\phi} p_{\phi}+G^{++} p_{++}-G^{--} p_{--}, \\
\tilde{H}_{(1)} & =G^{i} p_{i}
\end{aligned}
$$

even a linear algebra emerges that closes entirely under derivatives of $\delta$-functions and contains $G$ as a semi-direct product:

$$
\begin{aligned}
\left\{\tilde{H}_{(0)}, \tilde{H}_{(0)}^{\prime}\right\} & =\left(\tilde{H}_{(1)}+\tilde{H}_{(1)}^{\prime}\right) \partial \delta\left(x-x^{\prime}\right) \\
\left\{\tilde{H}_{(0)}, \tilde{H}_{(1)}^{\prime}\right\} & =\left(\tilde{H}_{(0)}+\tilde{H}_{(0)}^{\prime}\right) \partial \delta\left(x-x^{\prime}\right) \\
\left\{\tilde{H}_{(1)}, \tilde{H}_{(1)}^{\prime}\right\} & =\left(\tilde{H}_{(1)}+\tilde{H}_{(1)}^{\prime}\right) \partial \delta\left(x-x^{\prime}\right) \\
\left\{G, \tilde{H}_{(0)}^{\prime}\right\} & =G^{\prime} \partial \delta\left(x-x^{\prime}\right) \\
\left\{G, \tilde{H}_{(1)}^{\prime}\right\} & =G^{\prime} \partial \delta\left(x-x^{\prime}\right) \\
\left\{G, G^{\prime}\right\} & =0
\end{aligned}
$$

By defining the (anti-)holomorphic "currents" $\tilde{H}_{( \pm)}=\frac{1}{2}\left(\tilde{H}_{(0)} \pm \tilde{H}_{(1)}\right)$, (2.17) transforms into the standard Virasoro algebra of string theory. Thus, although the original PSM was formulated in terms of a non-linear $W$-algebra for the PSM constraints $G^{i}$, by this simple redefinition the deformation from the dilaton field is hidden in the non-trivial, but still linear, action of the abelian Lorentz-constraint $G$.

\section{Algebras for generic gPSM gravity}

The method of redefining constraints as in (2.13) or (2.16) shall now be extended to the graded version of the PSM, including fermionic generators $I_{( \pm)}$besides the ADM constraints $H_{( \pm)}$. An essential ingredient for a "genuine" supergravity is that it possesses rigid supersymmetry as a limiting case when the deformations are turned off and when the metric becomes the one describing flat Minkowski space. It is, therefore, imperative as a first step to study rigid supersymmetry in this formalism and then to consider deformations which agree with the basic principles set out in section 1. The choice of those requirements, of course, will be largely determined by the experience drawn from this particular case. 


\subsection{Rigid supersymmetry}

In the gPSM formulation rigid supersymmetry is produced by the specific choice of the Poisson tensor [38]

$$
\begin{gathered}
P^{a \phi}=X^{b} \epsilon_{b}{ }^{a}, \quad P^{\alpha \phi}=-\frac{1}{2} \chi^{\beta} \gamma_{\beta}^{3}{ }^{\alpha}, \\
P^{\alpha \beta}=\tilde{u}_{0} i X^{c} \gamma_{c}^{\alpha \beta}+c\left(\gamma^{3}\right)^{\alpha \beta}, \\
P^{a b}=0, \quad P^{a \beta}=0 .
\end{gathered}
$$

Here and in the following we restrict by the choice (3.2) to full $N=(1,1)$ supergravity ("full fermionic rank" of ref. [38]). Equations (3.1) are uniquely determined by the requirement of Lorentz covariance (cf. (A.8)). The first term in (3.2) is seen to produce the minimal contribution to the supertorsion, $c$ is an arbitrary constant. Indeed, inserting (3.1)-(3.3) into $(2.9)$ yields

$$
\begin{gathered}
\left\{G^{a}, G^{\phi}\right\}=-G^{b} \epsilon_{b}{ }^{a}, \quad\left\{G^{\alpha}, G^{\phi}\right\}=\frac{1}{2} G^{\beta} \gamma_{\beta}^{3}{ }^{\alpha}, \\
\left\{G^{\alpha}, G^{\beta}\right\}=-\tilde{u}_{0} i G^{a} \gamma_{a}^{\alpha \beta}, \\
\left\{G^{a}, G^{b}\right\}=0, \quad\left\{G^{a}, G^{\alpha}\right\}=0 .
\end{gathered}
$$

Choosing the normalization $\tilde{u}_{0}=2$ this is the well-known supersymmetry algebra ${ }^{9}$ with Lorentz transformations induced by the generator $G^{\phi}$, translations $G^{a}$ and supersymmetry transformations generated by $G^{\alpha}$.

Analogously to the non-supersymmetric case we now derive the superconformal algebra by an appropriate choice of new constraints. The fermionic constraint must be the "square root" of the Hamiltonian constraints, proportional to $G^{\alpha}=\left(G^{+}, G^{-}\right)$and, like $H_{(0)}$ and $H_{(1)}$, remain invariant under local Lorentz transformations $G^{\alpha} \rightarrow\left(e^{\lambda} G^{+}, e^{-\lambda} G^{-}\right), p_{a} \rightarrow$ $\left(e^{-2 \lambda} p_{++}, e^{2 \lambda} p_{--}\right)$. From these conditions it is easy to guess that $I_{(+)} \propto G^{+} \sqrt{p_{++}}, I_{(-)} \propto$ $G^{-} \sqrt{p_{--}}$. Indeed, in terms of the constraints of type (2.13)

$$
H_{(+)}=G^{++} p_{++}+G^{+} p_{+}, \quad H_{(-)}=-G^{--} p_{--}-G^{-} p_{-},
$$

supplemented by the fermionic constraints $\left(\tilde{u}_{0}=2\right)$

$$
I_{(+)}= \pm \frac{1}{\sqrt{2 \sqrt{2}}} G^{+} \sqrt{p_{++}}, \quad I_{(-)}= \pm \frac{1}{\sqrt{2 \sqrt{2}}} G^{-} \sqrt{p_{--}}
$$

$H_{( \pm)}$and $I_{( \pm)}$are found to generate the standard superconformal algebra, whereas the

\footnotetext{
${ }^{9}$ Notice our conventions for the $\gamma$ matrices in eqs. (A.6) and (A.12).
} 
Lorentz constraint decouples completely:

$$
\begin{aligned}
\left\{H_{(+)}, H_{(+)}^{\prime}\right\} & =\left(H_{(+)}+H_{(+)}^{\prime}\right) \partial \delta\left(x-x^{\prime}\right) \\
\left\{H_{(-)}, H_{(-)}^{\prime}\right\} & =-\left(H_{(-)}+H_{(-)}^{\prime}\right) \partial \delta\left(x-x^{\prime}\right) \\
\left\{H_{(+)}, H_{(-)}^{\prime}\right\} & =0 \\
\left\{I_{(+)}, I_{(+)}^{\prime}\right\} & =-2 H_{(+)} \delta\left(x-x^{\prime}\right) \\
\left\{I_{(-)}, I_{(-)}^{\prime}\right\} & =2 H_{(-)} \delta\left(x-x^{\prime}\right) \\
\left\{I_{(+)}, I_{(-)}^{\prime}\right\} & =0 \\
\left\{I_{(+)}, H_{(+)}^{\prime}\right\} & =\left(I_{(+)}+\frac{1}{2} I_{(+)}^{\prime}\right) \partial \delta\left(x-x^{\prime}\right) \\
\left\{I_{(-)}, H_{(-)}^{\prime}\right\} & =-\left(I_{(-)}+\frac{1}{2} I_{(-)}^{\prime}\right) \partial \delta\left(x-x^{\prime}\right) \\
\left\{I_{(-)}, H_{(+)}^{\prime}\right\} & =\left\{I_{(+)}, H_{(-)}^{\prime}\right\}=0 \\
\left\{H_{( \pm)}, G^{\prime}\right\} & =\left\{I_{( \pm)}, G^{\prime}\right\}=0
\end{aligned}
$$

From (3.7) we see that this algebra is of the type (2.13)-(2.15) rather than $(2.16)-(2.18)$.

Requirements (1) and (2) are fulfilled trivially, requirement (3) even holds without the Lorentz constraint $G^{\phi}=G$ appearing on the right hand side of (3.10). Replacing $H_{( \pm)}$by the alternative choice (2.16) $\tilde{H}_{(+)}=H_{(+)}+G^{\phi} p_{\phi}$ and $\tilde{H}_{(-)}=H_{(-)}$we arrive at

$$
\begin{aligned}
& \left\{I_{(+)}, I_{(+)}^{\prime}\right\}=-2\left(\tilde{H}_{(+)}-G^{\phi} p_{\phi}\right) \delta\left(x-x^{\prime}\right) \\
& \left\{I_{(-)}, I_{(-)}^{\prime}\right\}=2 \tilde{H}_{(-)} \delta\left(x-x^{\prime}\right) .
\end{aligned}
$$

Thus in contrast to (3.9)-(3.12) the algebra of rigid supersymmetry involving $\tilde{H}_{( \pm)}$is no longer linear.

\subsection{Algebra for generic gPSMs}

We now extend the considerations of the last subsection to general supergravity models. Before evaluating the "dilaton deformed" algebra we have to define a suitable extension of (3.7) (or its counterpart $\tilde{H}_{( \pm)}$) and (3.8). Here the second requirement of the introduction is essential: $H_{( \pm)}$should retain the structure as given in (3.7), whereas for the the fermionic generators the form (3.8) implies

$$
I_{(+)}= \pm a G^{+} \sqrt{p_{++}}, \quad I_{(-)}= \pm a G^{-} \sqrt{p_{--}} .
$$

Of course, for the most general form of $I_{( \pm)}$the ansatz $I_{( \pm)}= \pm a G^{ \pm} \sqrt{p_{ \pm \pm}}+\tilde{I}_{( \pm)}$could be made. $\tilde{I}_{( \pm)}$would be an arbitrary fermionic operator built from the variables in (2.8)(2.10). The limit of rigid supersymmetry would dictate $\tilde{I}_{( \pm)} \Rightarrow 0$. Now requirement (2) simply states that $\tilde{I}_{( \pm)} \equiv 0$ has been chosen. An analogous argument from requirement (2) precludes a further fermionic extension of $H_{( \pm)}$. Requirement (1) suggests $a=$ const. in (3.15), but we will have to check the consequences of $a(\phi, Y)$ as well later on. 
With the definitions (3.7), resp. (3.15) the general superconformal algebra, after a laborious but straightforward calculation, using (2.8)-(2.10) becomes

$$
\begin{aligned}
& \left\{H_{(+)}, H_{(+)}^{\prime}\right\}=\left(H_{(+)}+H_{(+)}^{\prime}\right) \partial \delta\left(x-x^{\prime}\right), \\
& \left\{H_{(-)}, H_{(-)}^{\prime}\right\}=-\left(H_{(-)}+H_{(-)}^{\prime}\right) \partial \delta\left(x-x^{\prime}\right) \text {, } \\
& \left\{H_{(+)}, H_{(-)}^{\prime}\right\}=G \partial_{\phi}\left(P^{--K} p_{K} p_{--}+P^{-K} p_{K} p_{-}\right) \delta\left(x-x^{\prime}\right) \text {, } \\
& \left\{I_{(+)}, I_{(+)}^{\prime}\right\}=-a^{2}\left(G^{+} \partial_{++} P^{+K} p_{K}+G^{K} \partial_{K} P^{+\mid+} p_{++}\right) \delta\left(x-x^{\prime}\right), \\
& \left\{I_{(-)}, I_{(-)}^{\prime}\right\}=-a^{2}\left(G^{-} \partial_{--} P^{-K} p_{K}+G^{K} \partial_{K} P^{-\mid-} p_{--}\right) \delta\left(x-x^{\prime}\right), \\
& \left\{I_{(+)}, I_{(-)}^{\prime}\right\}=-a^{2}\left(G^{+} \partial_{++} P^{-K} p_{K} \frac{\sqrt{p_{--}}}{2 \sqrt{p_{++}}}+G^{-} \partial_{--} P^{+K} p_{K} \frac{\sqrt{p_{++}}}{2 \sqrt{p_{--}}}\right. \\
& \left.+G^{K} \partial_{K} P^{+-} \sqrt{p_{++}} \sqrt{p_{--}}\right) \delta\left(x-x^{\prime}\right), \\
& \left\{I_{(+)}, H_{(+)}^{\prime}\right\}=\left(I_{(+)}+\frac{1}{2} I_{(+)}^{\prime}\right) \partial \delta\left(x-x^{\prime}\right) \\
& -\left( \pm \frac{a}{2}\right)\left(G \partial_{\phi} P^{+K} p_{K} \sqrt{p_{++}}-\mathcal{D}^{+}\right) \delta\left(x-x^{\prime}\right), \\
& \left\{I_{(-)}, H_{(-)}^{\prime}\right\}=-\left(I_{(-)}+\frac{1}{2} I_{(-)}^{\prime}\right) \partial \delta\left(x-x^{\prime}\right) \\
& +\left( \pm \frac{a}{2}\right)\left(G \partial_{\phi} P^{-K} p_{K} \sqrt{p_{--}}+\mathcal{D}^{-}\right) \delta\left(x-x^{\prime}\right), \\
& \left\{I_{(-)}, H_{(+)}^{\prime}\right\}=-\left( \pm \frac{a}{2}\right)\left(G \partial_{\phi} P^{-K} p_{K} \sqrt{p_{--}}-\mathcal{D}^{-}\right) \delta\left(x-x^{\prime}\right), \\
& \left\{I_{(+)}, H_{(-)}^{\prime}\right\}=\left( \pm \frac{a}{2}\right)\left(G \partial_{\phi} P^{+K} p_{K} \sqrt{p_{++}}+\mathcal{D}^{+}\right) \delta\left(x-x^{\prime}\right), \\
& \left\{H_{( \pm)}, G^{\prime}\right\}=\left\{I_{( \pm)}, G^{\prime}\right\}=0 ，
\end{aligned}
$$

where $\mathcal{D}^{ \pm}$in $\left\{I_{( \pm)}, H_{( \pm)}^{\prime}\right\}$ are abbreviations for

$$
\begin{aligned}
\mathcal{D}^{+}= & -\frac{1}{2} G^{+} \partial_{++}\left(P^{++K} p_{K} \sqrt{p_{++}}-P^{--K} \frac{p_{K} p_{--}}{\sqrt{p_{++}}}+P^{+K} \frac{p_{K} p_{+}}{\sqrt{p_{++}}}-P^{-K} \frac{p_{K} p_{-}}{\sqrt{p_{++}}}\right) \\
& -G^{K} \partial_{K}\left(P^{+\mid++} p_{++}-P^{+\mid--} p_{--}+P^{+\mid+} p_{+}-P^{+\mid-} p_{-}\right) \sqrt{p_{++}} \\
& +\left(G^{++} \partial_{++}-G^{--} \partial_{--}+G^{+} \partial_{+}-G^{-} \partial_{-}\right) P^{+K} p_{K} \sqrt{p_{++}} \\
\mathcal{D}^{-}= & -\frac{1}{2} G^{-} \partial_{--}\left(P^{++K} \frac{p_{K} p_{++}}{\sqrt{p_{--}}}-P^{--K} p_{K} \sqrt{p_{--}}+P^{+K} \frac{p_{K} p_{+}}{\sqrt{p_{--}}}-P^{-K} \frac{p_{K} p_{-}}{\sqrt{p_{--}}}\right) \\
& -G^{K} \partial_{K}\left(P^{-\mid++} p_{++}-P^{-\mid--} p_{--}+P^{-\mid+} p_{+}-P^{-\mid-} p_{-}\right) \sqrt{p_{--}} \\
& +\left(G^{++} \partial_{++}-G^{--} \partial_{--}+G^{+} \partial_{+}-G^{-} \partial_{-}\right) P^{-K} p_{K} \sqrt{p_{--}}
\end{aligned}
$$

The components of the Poisson tensor $P^{I J}$ in (3.16)-(3.19) are still completely general, except for those dictated by local Lorentz transformations (cf. eqs. (3.1) and (3.4)). Comparing with (3.9)-(3.12) we note that the deformation of the algebra does not lead to 
additional structure functions $\propto \partial \delta$, which happens to be a consequence of our choice of requirement (2). Obviously in supergravity no simple redefinition of the constraints can lead to an algebra including structure constants only instead of structure functions. The main obstacle is the fact that any redefinition of the constraints $H_{( \pm)}$, simplifying the purely bosonic part of the algebra, inevitably leads to new non-linear terms in the anti-commutator of two spinorial generators.

So far $a$ in equation (3.15) has been assumed to be constant. That this is in agreement with requirement (1) can be seen from the origin of that constant. In the general decomposition ${ }^{10}$ of $P^{\alpha \beta}$

$$
P^{\alpha \beta}=v^{\alpha \beta}+\frac{1}{2} \chi^{2} v_{2}^{\alpha \beta}
$$

in the term proportional to $\gamma_{c}$ in

$$
v^{\alpha \beta}=i \tilde{u} X^{c} \gamma_{c}^{\alpha \beta}+u \gamma^{3 \alpha \beta},
$$

requirement (1) at the gPSM level, comparing with the case of rigid supersymmetry (3.2), suggests to take $\tilde{u}=\tilde{u}_{0}=\left(\sqrt{2} a^{2}\right)^{-1}$ to be constant. Indeed $a$ so far has been chosen in that way.

One should, nevertheless, check the consequences of relaxing this restriction and allowing $a$ in (3.15) to become a function of the dilaton field $\phi$ and of $Y=X^{a} X_{a} / 2$. We first treat $a=a(\phi)$. Then additional deformations emerge in certain commutators among (3.16)-(3.19):

$$
\begin{aligned}
\left\{I_{(+)}, I_{(-)}^{\prime}\right\} & =( \pm) \partial_{\phi} a\left(P^{-\phi} \sqrt{p_{--}} I_{(+)}-P^{+\phi} \sqrt{p_{++}} I_{(-)}\right) \delta\left(x-x^{\prime}\right)+\ldots \\
\left\{I_{(+)}, H_{(+)}^{\prime}\right\} & =-( \pm) \partial_{\phi} \ln a\left(P^{++\phi} p_{++}+P^{+\phi} p_{+}\right) I_{(+)} \delta\left(x-x^{\prime}\right)+\ldots \\
\left\{I_{(+)}, H_{(-)}^{\prime}\right\} & =( \pm) \partial_{\phi} \ln a\left(P^{--\phi} p_{--}+P^{-\phi} p_{-}\right) I_{(+)} \delta\left(x-x^{\prime}\right)+\ldots \\
\left\{I_{(-)}, H_{(+)}^{\prime}\right\} & =-( \pm) \partial_{\phi} \ln a\left(P^{++\phi} p_{++}+P^{+\phi} p_{+}\right) I_{(-)} \delta\left(x-x^{\prime}\right)+\ldots \\
\left\{I_{(-)}, H_{(-)}^{\prime}\right\} & =( \pm) \partial_{\phi} \ln a\left(P^{--\phi} p_{--}+P^{-\phi} p_{-}\right) I_{(-)} \delta\left(x-x^{\prime}\right)+\ldots
\end{aligned}
$$

The commutators $\left\{I_{(+)}, I_{(+)}^{\prime}\right\}$ and $\left\{I_{(-)}, I_{(-)}^{\prime}\right\}$ do not experience any change. If $a$ becomes a function of $Y,\left\{I_{(+)}, I_{(+)}^{\prime}\right\}$ receives additional contributions

$$
\left\{I_{(+)}, I_{(+)}^{\prime}\right\}=-a P^{+c} \partial_{c} a G^{+} p_{++} \delta\left(x-x^{\prime}\right) .
$$

The right hand side of (3.25) cannot be made a contribution to the Hamiltonian (3.7) as dictated for this anti-commutator by requirement (3). The only other possibility would be $P^{+c}=0$. But this restriction would lead back to the special case of rigid supersymmetry ${ }^{11}$. Thus $\tilde{u}=\tilde{u}(Y)$ for any non-trivial algebra would always contradict requirement (3). If

\footnotetext{
${ }^{10}$ For full fermionic rank, i.e. $\operatorname{det} v^{\alpha \beta} \neq 0$ only $\gamma_{c}$ and $\gamma^{3}$ are allowed (cf. after eq. A.6)). The Majorana nature of $\chi$ forbids terms $\mathcal{O}\left(\chi^{3}\right)$.

${ }^{11}$ A different Poisson tensor ("block diagonal supergravity") with $P^{+c}=0$ had been presented in [38]. But in that case the most general $P^{\alpha \beta}$ has the form $P^{\alpha \beta}=u(\phi, Y)\left(\gamma^{3}\right)^{\alpha \beta}$, precluding the interpretation of the fermionic $\epsilon_{\alpha}$ as supersymmetry transformations.
} 
$\tilde{u}=\tilde{u}(\phi, Y)$ both deformations (3.24) and (3.25) add. Thus from now on only a dependence $\tilde{u}=\tilde{u}(\phi)$ will be permitted, because an $Y$-dependence has been excluded.

The absence of the Lorentz constraint $G$ on the r.h.s of $\left\{I_{(+)}, I_{(+)}^{\prime}\right\}$ and $\left\{I_{(-)}, I_{(-)}^{\prime}\right\}$ for general $\tilde{u}=\tilde{u}(\phi, Y)$ implies that the third requirement of section 11 can be tightened for the choice of $H_{( \pm)}$as in (3.7):

(3a) The constraints $I_{(+)}$with itself and $I_{(-)}$with itself anti-commute back into $H_{(0)}$ and $H_{(1)}$.

Still the general structure of (3.16)-(3.21) shows the possibility of singularities at $p_{++}=$ $e_{1 \mid++}=0$ and $p_{--}=e_{1 \mid--}=0$ (cf. eq. (2.2)). These singularities will turn out to disappear when the content of requirement (3) of the introduction (resp. (3a) above) is taken into account in full mathematical detail and not only qualitatively, as has been done so far.

One could be tempted to pursue the complete procedure of this section by trying to simplify the algebra by the use of $\tilde{H}_{( \pm)}$instead of $H_{( \pm)}$. However, it can be seen easily that one would be forced to weaken the supersymmetry relation of the Hamiltonian constraints, being the "square" of the supersymmetry generators. It turns out that this does not remove

the complicated structure of $\left\{I_{( \pm)}, H_{( \pm)}^{\prime}\right\}$, in particular it does not remove the dependence of these commutators on the constraints $G^{a}$ and $G^{\alpha}$.

It may be useful to summarize the results obtained so far: As a consequence of requirements (1) and (2) the Hamiltonian constraints are (3.7), while the fermionic ones must be chosen as in (3.15), the latter being suggested by the special case of rigid supersymmetry. The dependence of the factor $a=(\sqrt{2} \tilde{u})^{-1 / 2}$ on $Y$ is forbidden, but it still may depend on $\phi$ at this point. Only an analytic implementation of requirement (3) in section 5 will show that, at the end of the day a constant $a$, resp. $\tilde{u}=\tilde{u}_{0}$ alone will be allowed.

\section{Dilaton prepotential algebra: a special example}

In order to check whether the three requirements are satisfied by some gPSM model with non-trivial gravity sector, we illustrate the above algebra by an important example, the dilaton prepotential algebra. This model originally had been studied in ref. [47] within a different approach, a derivation in terms of graded Poisson Sigma Models has been given in ref. [38]. As the bosonic potential is restricted here to be a function of the dilaton field $\phi$ only, these models have vanishing bosonic torsion $(Z=0$ in (1.6)). For a Poisson tensor, which shares the first term in (4.3) with the one in rigid supersymmetry (3.2), the solution of (1.2) has been given in ref. [38], eqs. (5.34)-(5.36) $\left(u^{\prime}=\partial u / \partial \phi\right)$

$$
\begin{aligned}
P^{a b} & =\frac{1}{2 \tilde{u}_{0}^{2}}\left(-\left(u^{2}\right)^{\prime}+\frac{1}{2} \chi^{2} u^{\prime \prime}\right) \epsilon^{a b}, \\
P^{\alpha b} & =\frac{i u^{\prime}}{2 \tilde{u}_{0}}\left(\chi \gamma^{b}\right)^{\alpha}, \\
P^{\alpha \beta} & =i \tilde{u}_{0} X^{c} \gamma_{c}^{\alpha \beta}+u \gamma^{3 \alpha \beta},
\end{aligned}
$$


where $\tilde{u}_{0}=$ const. and $u(\phi)$ is a "prepotential" related to the bosonic potential $V$ in (1.6) as (cf. eq. (5.30) of ref. [38])

$$
\tilde{u}_{0}^{2} V+u u^{\prime}=0
$$

In terms of (3.1) and (4.1)- (4.3) the gPSM action (1.1)

$$
\begin{aligned}
\mathcal{S}_{D P P}=\int_{\mathcal{M}}\left(\phi \mathrm{d} \omega+X^{a} D e_{a}+\chi^{\alpha} D \psi_{\alpha}\right. & +\epsilon\left(V-\frac{1}{4 u} \chi^{2}\left(V^{\prime}+4 \frac{V^{2}}{u^{2}}\right)\right) \\
& \left.+\frac{i V}{u}\left(\chi \gamma^{a} e_{a} \psi\right)+i X^{a}\left(\psi \gamma_{a} \psi\right)-\frac{1}{2} u\left(\psi \gamma_{3} \psi\right)\right)
\end{aligned}
$$

with the covariant derivatives (A.8) includes the standard contribution to the supertorsion from the fermions only, as can be observed from the factor of $X^{a}$. This class of models already covers the supersymmetrized version of several known two-dimensional gravity models ${ }^{12}$, in particular (1.7) and (1.8). Eliminating [38] algebraically the auxiliary fields $X^{a}$ and $\omega$ in (4.5) one arrives at a superdilaton action of the form

$$
\begin{aligned}
\mathcal{S}_{D P P}=\int \mathrm{d}^{2} x e\left(\frac{1}{2} \tilde{R} \phi+(\chi \tilde{\sigma})+V-\frac{1}{4 u} \chi^{2}\right. & \left(V^{\prime}+4 \frac{V^{2}}{u^{2}}\right) \\
& \left.-\frac{i V}{u} \epsilon^{m n}\left(\chi \gamma_{n} \psi_{m}\right)+\frac{u}{2} \epsilon^{m n}\left(\psi_{n} \gamma^{3} \psi_{m}\right)\right) .
\end{aligned}
$$

The Ricci scalar $\tilde{R}=2 * \mathrm{~d} \tilde{\omega}$ is expressed in terms of the dependent spin connection

$$
\tilde{\omega}_{a}=\epsilon^{m n} \partial_{n} e_{m a}-i \epsilon^{m n}\left(\psi_{n} \gamma_{a} \psi_{m}\right)
$$

Its fermionic counterpart is

$$
\tilde{\sigma}_{\alpha}=*(\tilde{D} \psi)_{\alpha}=\epsilon^{m n}\left(\partial_{n} \psi_{m \alpha}+\frac{1}{2} \tilde{\omega}_{n}\left(\gamma^{3} \psi_{m}\right)_{\alpha}\right) .
$$

$V$ and $u$ are still related by (4.4). This new form of the action turns out to be a special case of the superdilaton action obtained from superspace as introduced by Park and Strominger [39], corresponding to $J(\Phi)=\Phi$ and $K(\Phi)=0$ in the notation of ref. [39]. The relation between the dilaton supergravity by Park and Strominger and this special case following from a gPSM has been observed in ref. [51], a more detailed discussion of this topic will be given in [52]. In addition this model can be related to the supergravity model of Howe quite generally $[38,52]$.

Together with the relations (3.4) the constraint algebra (2.9) for (4.1)-(4.3) becomes

$$
\begin{aligned}
\left\{G^{a}, G^{b}\right\} & =-\frac{\epsilon^{a b}}{2 \tilde{u}_{0}^{2}}\left(G^{\phi}\left(-\left(u^{2}\right)^{\prime \prime}+\frac{1}{2} \chi^{2} u^{\prime \prime \prime}\right)+G^{\alpha} \chi_{\alpha} u^{\prime \prime}\right), \\
\left\{G^{\alpha}, G^{b}\right\} & =-\frac{i}{2 \tilde{u}_{0}}\left(G^{\phi} u^{\prime \prime}\left(\chi \gamma^{b}\right)^{\alpha}+G^{\beta}\left(\gamma^{b}\right)_{\beta}{ }^{\alpha}\right), \\
\left\{G^{\alpha}, G^{\beta}\right\} & =-i \tilde{u}_{0} G^{a} \gamma_{a}^{\alpha \beta}-G^{\phi} u^{\prime} \gamma^{3^{\alpha \beta}} .
\end{aligned}
$$

\footnotetext{
${ }^{12}$ The "topological" supergravity studied some time ago $[49,50]$ also may be interpreted as a similar gPSM with vanishing prepotential. On the other hand, it contains further generators from a central extension of the algebra.
} 
After a straightforward calculation the corresponding superconformal algebra is obtained in terms of $H_{( \pm)}$and $I_{( \pm)}$, which according to requirement (2) are given by eqs. (3.7) and (3.15):

$$
\begin{aligned}
&\left\{H_{(+)}, H_{(+)}^{\prime}\right\}=\left(H_{(+)}+H_{(+)}^{\prime}\right) \partial \delta\left(x-x^{\prime}\right) \\
&\left\{H_{(-)}, H_{(-)}^{\prime}\right\}=-\left(H_{(-)}+H_{(-)}^{\prime}\right) \partial \delta\left(x-x^{\prime}\right) \\
&\left\{H_{(+)}, H_{(-)}^{\prime}\right\}= G\left(\frac{1}{2 \tilde{u}_{0}^{2}}\left(\left(u^{2}\right)^{\prime \prime}+q^{+} q^{-} u^{\prime \prime \prime}\right) p_{++} p_{--}\right. \\
&\left.\quad-\frac{u^{\prime \prime}}{\sqrt{2} \tilde{u}_{0}}\left(q^{-} p_{+} p_{--}+q^{+} p_{-} p_{++}\right)-u^{\prime} p_{+} p_{-}\right) \delta\left(x-x^{\prime}\right) \\
&\left\{I_{(+)}, I_{(+)}^{\prime}\right\}=-2 H_{(+)} \delta\left(x-x^{\prime}\right) \\
&\left\{I_{(-)}, I_{(-)}^{\prime}\right\}= 2 H_{(-)} \delta\left(x-x^{\prime}\right) \\
&\left\{I_{(+)}, I_{(-)}^{\prime}\right\}= \pm \frac{\sqrt{2} i}{\tilde{u}_{0}} G u^{\prime} \sqrt{p_{++}} \sqrt{p_{--}} \delta\left(x-x^{\prime}\right) \\
&\left\{I_{(+)}, H_{(+)}^{\prime}\right\}=\left(I_{(+)}+\frac{1}{2} I_{(+)}^{\prime}\right) \partial \delta\left(x-x^{\prime}\right) \\
&\left\{I_{(-)}, H_{(-)}^{\prime}\right\}=-\left(I_{(-)}+\frac{1}{2} I_{(-)}^{\prime}\right) \partial \delta\left(x-x^{\prime}\right) \\
&\left\{I_{(-)}, H_{(+)}^{\prime}\right\}= \pm \sqrt{\frac{\sqrt{2}}{\tilde{u}_{0}}} G\left(\frac{u^{\prime \prime}}{\sqrt{2} \tilde{u}_{0}} q^{+} p_{++}+u^{\prime} p_{+}\right) \sqrt{p_{--}} \delta\left(x-x^{\prime}\right) \\
&\left\{I_{(+)}, H_{(-)}^{\prime}\right\}= \pm \sqrt{\frac{\sqrt{2}}{\tilde{u}_{0}}} G\left(\frac{u^{\prime \prime}}{\sqrt{2} \tilde{u}_{0}} q^{-} p_{--}+u^{\prime} p_{-}\right) \sqrt{p_{++}} \delta\left(x-x^{\prime}\right) \delta\left(x-x^{\prime}\right)
\end{aligned}
$$

Evidently the deformation is restricted to the appearance of the Lorentz constraint $\mathrm{G}$ which only occurs in the "mixed" (anti-)commutators of $\left\{H_{(+)}, H_{(-)}\right\},\left\{I_{(+)}, I_{(-)}\right\},\left\{I_{(-)}, H_{(+)}\right\}$ and $\left\{I_{(+)}, H_{(-)}\right\}$. Clearly the algebra obeys all the requirements set out in section 1 . Thus the identification of this model with others obtained from standard supergravity requirements [8-11,42] imply an important a posteriori confirmation of our requirements, as far as they have been taken into account already. However, our requirements will not only allow this special case. They will identify a more general class of gPSM derived theories with $Z \neq 0$ in (1.6), whose relation to models obtained from superspace has not been demonstrated so far in the literature.

Writing down the algebra in terms $\tilde{H}_{( \pm)}$of eqs. (2.16) does not provide any new insight into the structure of this theory. In contrast to the bosonic case (2.17) the undeformed superconformal algebra is found for constant $u$ only, which corresponds to rigid supersymmetry.

In view of the singularity problems of a generic gPSM [38] it is important to study the regularity of the algebra about which no assumptions have been made so far. An obvious constraint is $\tilde{u}_{0} \neq 0$, which however is irrelevant from the physical point of view: For $\tilde{u}_{0}=0$ supersymmetry transformations are no longer generated by $G^{\alpha}$ and thus the meaning of 
the model is lost. Furthermore in contrast to the general algebra (3.16)-(3.19), the dilaton prepotential supergravity is regular for $p_{a}=0$, which, in this particular case, is easily seen to be a trivial consequence of vanishing bosonic torsion: all potentially singular terms in eqs. (3.17), (3.20) and (3.21) are multiplied by vanishing derivatives $\partial_{c} P^{\alpha a}$ and $\partial_{c} P^{+\mid-}$.

\section{General deformed superconformal algebra}

We now show that the most general supergravity model described by a gPSM, obeying the restrictions of section 1, must exhibit a similar structure as the dilaton prepotential algebra of section 4 . The only possible generalization will turn out to be closely related to the algebra (4.12)-(4.14) as well, because it may be produced by a special target space diffeomorphism from the latter.

The requirements (1) and (2) are implemented already in (3.16)-3.19). Starting from the most general gPSM algebra (3.16)-(3.19) we now demand the anti-commutators $\left\{I_{(+)}, I_{(+)}^{\prime}\right\}$ and $\left\{I_{(-)}, I_{(-)}^{\prime}\right\}$ to yield exactly the Hamiltonian $H_{( \pm)}$(requirement (3a)).

Comparison of the right hand side of the first two equations of $(3.17)^{13}$ with the desired expression for $H_{( \pm)}$(cf. (3.7)) translates into six necessary and sufficient conditions on the Poisson tensor:

$$
\begin{aligned}
& \frac{\partial}{\partial X^{++}}\left(P^{+\mid-}, P^{+\mid++}, P^{+\mid--}\right)=0 \\
& \frac{\partial}{\partial X^{--}}\left(P^{+\mid-}, P^{-\mid--}, P^{-\mid++}\right)=0
\end{aligned}
$$

As an example we consider the r.h.s. of the first equation of (3.17). Due to the restrictions that followed from the discussion in section 3.2, the second term of that expression can contribute with an expression of the form $G^{++} p_{++}$only, which is part of $H_{(+)}$. The first term in the first eq. of (3.17), however, contributes $G^{+} p_{++}, G^{+} p_{--}$and $G^{+} p_{-}$, which do not appear in $H_{(+)}$. They are eliminated by the first three conditions in (5.1).

In the following we need the general explicit solution of the vanishing graded Nijenhuis tensor (1.2) already obtained in ref. [38]. As we do not want to repeat the lengthy calculation leading to this result, we refer the reader for all notations and details of the calculation to this work, especially sections 3,5.4 and 5.5. If the technical details of the detailed implementation of (5.1) are not interesting to the reader, he/she may jump to the final result as given in (5.15)-(5.17) together with (5.11) below. Within the given approach this represents the most general Poisson tensor describing supergravity.

The argument starts with the case $\tilde{u}=\tilde{u}_{0}=$ constant. Applying (5.1) to the general decomposition for $P^{\alpha \beta}$ of eqs. (3.22) and (3.23) restricts its terms as

$$
v^{\alpha \beta}=i \tilde{u}_{0} X^{c} \gamma_{c}^{\alpha \beta}+u(\phi) \gamma^{3^{\alpha \beta}}, \quad v_{2}^{\alpha \beta}=\tilde{v}(\phi) \gamma^{3^{\alpha \beta}} .
$$

The bosonic part $P^{a b}$ is still general (cf. [38])

$$
P^{a b}=\epsilon^{a b}\left(v(\phi, Y)+\frac{1}{2} \chi^{2} v_{2}(\phi, Y)\right) .
$$

\footnotetext{
${ }^{13}$ The comment immediately after eq. (3.24) is important here.
} 
The first term in (5.3) is the input from the bosonic theory (1.5), (1.6), the second is the only possible one in presence of the dilatino. As shown in ref. [38] the complete solution from the vanishing Nijenhuis tensor is parametrized by $v^{\alpha \beta}, v$ and a Lorentz vector field $f^{a}$, which appears in the decomposition of the "mixed" component of the Poisson tensor

$$
P^{\alpha b}=\chi^{\beta} F_{\beta}^{b}{ }^{\alpha} .
$$

Together with other terms it creates a contribution to the decomposition (5.4) of the form

$$
F_{\alpha}^{c}{ }^{\beta}=i f(\phi) X^{c} X^{b} \gamma_{b \alpha}{ }^{\beta}+\ldots,
$$

with an invariant function $f(\phi)$. In ref. [38] in order to eliminate the term (5.5) the special choice $f^{a}=\frac{1}{2} u^{\prime} X^{a}$ has been made. In that reference this was motivated by the desire to present a simple solution and, at the same time, to make contact with an earlier result of ref. [47]. In our present context a term like (5.5), producing in (5.4) contributions to $P^{\alpha a}$ of type

$$
P^{+\mid++} \sim X^{++} X^{++} \chi^{-}+\ldots, \quad P^{+\mid--} \sim X^{--} X^{++} \chi^{-}+\ldots
$$

would be in obvious contradiction to (5.1). Thus the same choice of $f^{a}$ as in ref. [38] must be made. It should be noted that the remaining terms in $F_{\alpha}^{c}{ }^{\beta}$ yield $P^{++++} \equiv 0$.

Due to the restrictions from (5.1) found so far according to (5.2) and (5.5), $v_{2}^{\alpha \beta}$ in (5.2) and $v_{2}$ in (5.3) can be parametrized in terms of two remaining functions of the dilaton field: the bosonic potential $v(\phi)$ and the prepotential $u(\phi)$. As this specific solution has been selected for a quite different reason already in ref. [38] (cf. above) we are allowed to take over the final result from there (eqs. (5.27)-(5.29) in [38]):

$$
\begin{aligned}
F_{\alpha}^{c}{ }^{\beta} & =\frac{1}{2 \Delta}\left(\tilde{u}_{0}^{2} v+u u^{\prime}\right) X^{a}\left(\gamma_{a} \gamma^{c} \gamma^{3}\right)_{\alpha}^{\beta}+\frac{i \tilde{u}_{0}}{2 \Delta}\left(u v+2 Y u^{\prime}\right) \gamma_{\alpha}^{c \beta}, \\
v_{2}^{\alpha \beta} & =\frac{1}{2 \Delta}\left(\tilde{u}_{0}^{2} v+u u^{\prime}\right) \gamma^{3^{\alpha \beta}}, \\
v_{2} & =\frac{u v}{2 \Delta^{2}}\left(\tilde{u}_{0}^{2} v+u u^{\prime}\right)+\frac{u u^{\prime}}{2 \Delta^{2}}\left(u v+2 Y u^{\prime}\right)+\frac{1}{2 \Delta}\left(u v^{\prime}+2 Y u^{\prime} \dot{v}+2 Y u^{\prime \prime}\right),
\end{aligned}
$$

with

$$
\Delta=2 Y \tilde{u}_{0}^{2}-u^{2}
$$

being the determinant of $v^{\alpha \beta}$. Derivatives with respect to the dilaton field are indicated by a prime, derivatives with respect to $Y$ by a dot.

So far the function $u$ in the solution and the bosonic potential $v$ were not related. This residual freedom can now be used to achieve the cancellation of the $X^{a}$-dependence as required by (5.1) also for the components of the Poisson tensor still generated by (5.7) and (5.8). Starting from (5.8), $\partial_{++} P^{+\mid-}=0$ and noting that $X^{a}$ only appears in the $Y$-dependence of $v$ and $\Delta$ implies that the factor of $\gamma^{3}$ in (5.8) must be a function of $\phi$ alone. Therefore, a potential linear in $Y$ of (1.6) turns out to be the only allowed one! The ensuing relation

$$
V(\phi)=-\frac{\left(u^{2}\right)^{\prime}}{2 \tilde{u}_{0}^{2}}-\frac{u^{2}}{2 \tilde{u}_{0}^{2}} Z(\phi),
$$


with the definition of $\Delta$ may be rewritten as

$$
v=-\frac{\left(u^{2}\right)^{\prime}}{2 \tilde{u}_{0}}+\frac{1}{2 \tilde{u}_{0}^{2}} Z(\phi) \Delta
$$

However, adopting (5.12) for $v$ is found to eliminate at the same time all singularities $\propto \Delta^{-1}$ also in (5.7) and (5.9). The only remaining condition from (5.1), $\partial_{++} P^{+\mid--}=0$, is fulfilled automatically.

The divergence problem of (5.7)-(5.9) at $\Delta=0$ had been discussed extensively in ref. [38]. Therein the cancellations of these divergences was introduced as an ad hoc assumption and the most general function $v$ leading to that cancellation was found to be

$$
v=-\frac{\left(u^{2}\right)^{\prime}}{2 \tilde{u}_{0}}+T(\Delta, \phi, Y)
$$

with $\frac{T(\Delta, \phi, Y)}{\Delta}$ regular at $\Delta=0$. Eq. (5.12), obtained here by a different route, is a special case of that.

The fact that the new poles at $\Delta=0$ vanish at the level of the Poisson tensor is another confirmation of the appropriateness of our conditions (1) to (3) of section 1 (resp. (3a) of section [3). However, at first sight singularities at $p_{ \pm \pm}=0$ seem to persist at the level of the algebra (3.16)-(3.19). Inspecting the structure of the remaining deformations in the general algebra, it turns out that they disappear if besides (5.1) in addition

$$
\partial_{++} P^{++\mid--}=2 \partial_{+} P^{+\mid--}, \quad \partial_{++} P^{++\mid-}=2 \partial_{+} P^{+\mid-}
$$

holds, a condition which is indeed met in all models allowed by our requirements. To see this eq. (5.12) is used in (5.7)-(5.9) to eliminate $\Delta$. Then the the components $(3.22),(5.3)$ and (5.4) of the Poisson tensor become

$$
\begin{aligned}
P^{a b} & =\left(V+Y Z-\frac{1}{2} \chi^{2}\left(\frac{V Z+V^{\prime}}{2 u}+\frac{\tilde{u}_{0}^{2} V^{2}}{2 u^{3}}\right)\right) \epsilon^{a b} \\
P^{\alpha b} & =\frac{Z}{4} X^{a}\left(\chi \gamma_{a} \gamma^{b} \gamma^{3}\right)^{\alpha}-\frac{i \tilde{u}_{0} V}{2 u}\left(\chi \gamma^{b}\right)^{\alpha}, \\
P^{\alpha \beta} & =i \tilde{u}_{0} X^{c} \gamma_{c}^{\alpha \beta}+\left(u+\frac{Z}{8} \chi^{2}\right) \gamma^{3 \alpha \beta} .
\end{aligned}
$$

It can be checked that the factors in front of the expressions involving $Z$ exactly guarantee that the conditions (5.14) are satisfied. We conclude that our original conditions together with the symmetries of the Poisson tensor were also sufficient to ensure a regular superconformal algebra at $p_{ \pm \pm}=0$.

At the level of the models discussed so far $\left(\tilde{u}=\tilde{u}_{0}=\right.$ constant $)$ we observe that the following three conditions are equivalent:

(A) $\left\{I_{+}, I_{+}^{\prime}\right\}$ and $\left\{I_{-}, I_{-}^{\prime}\right\}$ have no deformations, which agrees with requirement (3) of section 1 .

(B) All deformations with respect to a superconformal algebra depend on the Lorentz constraint only. 
(C) The fermionic extensions of the Poisson tensor are regular at $\Delta=0$ and the algebra is regular at $p_{ \pm \pm}=0$.

Condition (B) obviously implies condition (A), but it is a remarkable result that our original condition is actually sufficient. We have shown above that condition (A) implies condition (C). To see that the reverse is true as well it is important to notice that we have to cancel the $f_{(t)}$ term in (5.5), although $P^{+\mid++}$now must be independent of $X^{--}$and not of $X^{++}$.

A final argument is required with respect to a dependence $\tilde{u}_{0} \rightarrow \tilde{u}(\phi)$ in (5.2), which is still an open possibility. This type of algebra has been studied in ref. [38] as well. Choosing again the independent vector field $f^{a}$ to obtain a cancellation of (5.5), (5.7) in that case generalizes to $\left(\nabla=\partial_{\phi}-v \partial_{Y}\right)$

$$
F^{c}=-\frac{1}{4}(\nabla \ln \Delta) X^{a}\left(\gamma_{a} \gamma^{c} \gamma^{3}\right)+\frac{1}{2}(\nabla \ln \tilde{u}) X^{c} \gamma^{3}+\frac{i \tilde{u} u}{2 \Delta}\left(v+2 Y\left(\nabla \ln \frac{u}{\tilde{u}}\right)\right) \gamma^{c} .
$$

Here both $u$ (note that the argument leading from (3.22) to (5.2) is still valid) and $\tilde{u}$ (cf. (3.25) ) are still allowed to depend on the dilaton field only. But when $\partial_{\phi} \tilde{u} \neq 0$ the term $\propto \gamma^{3}$ does not vanish and this leads to $X^{c}$-dependent contributions to $P^{+\mid++}$and $P^{-\mid--}$ which contradict (5.1). It should be noted that they are never able to cancel together with the other contributions to this part of the tensor, which we have set to zero by means of the independent vector field $f^{a}$. Thus only $\tilde{u}=\tilde{u}_{0}=$ const. is allowed by (5.1) and (5.15)-(5.17) is indeed the most general model obeying condition (A), resp. (3a).

Although (A) and (B) are still equivalent there exist some models with $\tilde{u}=\tilde{u}(\phi)$ that obey the condition (C) without fulfilling (A) and (B). An example of this type has been given in ref. [38], where (in section 5.7.1) a supersymmetric extension of spherically reduced gravity not fulfilling (A) and (B) can be found.

A more general $\tilde{u}(\phi, Y)$ already has been excluded in section 3.2 (cf. (3.25)). It is remarkable that a non-constant $\tilde{u}=\tilde{u}(\phi, Y)$ is not compatible too with the constraints (5.1) on the Poisson tensor. Indeed $\tilde{u}(Y)$ independent of the dilaton field is excluded as well, as $v \equiv 0$ is not a solution in this case. It remains the possibility of $\tilde{u}(\phi, Y)$ with $\nabla \tilde{u}=0$. To cancel all $Y$-dependent terms in $F^{c}$ which disagrees with (5.1) we would have to choose $v=-2 Y \frac{u^{\prime}}{u}$. But with that $v$ one finds that $v_{2}^{\alpha \beta}$ cannot be independent of $Y$ as well.

Starting from a given bosonic potential (1.6), the main restriction to the application of a "genuine" supergravity extension turns out to be (5.11). It is important to note that spherically reduced gravity (1.7) fits into this structure as do the models (1.8) and (1.9), but a model quadratic in torsion and curvature $[53,54]$ for certain values of its parameters does not in general, or, at best, experiences restrictions on the range of its bosonic fields.

As shown in ref. [38] the class of models determined by (5.12), resp. (5.11) can be obtained also by a conformal transformation of a dilaton prepotential model of section 4 , which may be interpreted as a special target space diffeomorphism of a gPSM. All this is a strong indication that among the gPSM models the ones with tensor (5.15)-(5.17) and the prepotential determined by the solution of (5.11) represent the most general "genuine" $N=(1,1)$ supergravity in $d=2$. The ensuing disappearance of the singularities in $\Delta$ and $p_{ \pm \pm}$in our approach is a fortuitous but welcome "accident". According to the solution of 
a general gPSM in [38] not obeying our requirements, generic singularities at $Y=0$ may be expected as well. Also those are absent here.

\section{General deformed supergravity}

Having shown that the most general supergravity model is the supersymmetrization of a bosonic model with a potential $V$ linear in $Y$ (cf. (1.6), but note the prepotential type restriction (5.11) on $V$ !) its (deformed) superalgebra can now be given explicitly. We economize writing it down in full detail by the observation that it shares most of its structure with the one of the dilaton prepotential algebra (4.12)-(4.14):

- The commutators of the form $\left\{A_{(+)}, B_{(+)}\right\}$and $\left\{A_{(-)}, B_{(-)}\right\}$exactly reproduce the non-trivial part of the well-known superconformal algebra. All deformations are in the commutators of the form $\left\{A_{(+)}, B_{(-)}\right\}$. This property is not difficult to read off from (3.16)-(3.19): $\left\{I_{(+)}, I_{(+)}^{\prime}\right\}$ and $\left\{I_{(-)}, I_{(-)}^{\prime}\right\}$ have been arranged such that they precisely yield $H_{(+)}$and $H_{(-)}$. For $\left\{I_{(+)}, H_{(+)}^{\prime}\right\}$ and $\left\{I_{(-)}, H_{(-)}^{\prime}\right\}$ one simply observes that the conditions imposed on the Poisson tensor directly lead to the last two equations of (3.18) with the much simpler quantities

$$
\begin{aligned}
& \mathcal{D}^{+}=G \partial_{\phi}\left(P^{+\mid--} p_{--}+P^{+\mid-} p_{-}\right) \sqrt{p_{++}}, \\
& \mathcal{D}^{-}=-G \partial_{\phi}\left(P^{-\mid++} p_{++}+P^{+\mid-} p_{+}\right) \sqrt{p_{--}} .
\end{aligned}
$$

- The deformation in the remaining commutators is a slight generalization of the dilaton prepotential algebra of section $4\left(a=\left(\sqrt{2} \tilde{u}_{0}\right)^{-1 / 2}\right)$ :

$$
\begin{aligned}
&\left\{H_{+}, H_{-}^{\prime}\right\}=G \partial_{\phi}\left(P^{--\mid++} p_{++} p_{--}+P^{--\mid+} p_{+} p_{--}\right. \\
&\left.+P^{-\mid++} p_{++} p_{-}+P^{+\mid-} p_{+} p_{-}\right) \delta\left(x-x^{\prime}\right) \\
&\left\{I_{+}, I_{-}^{\prime}\right\}=-a^{2} G \partial_{\phi} P^{+\mid-} \sqrt{p_{++}} \sqrt{p_{--}} \delta\left(x-x^{\prime}\right) \\
&\left\{I_{+}, H_{-}^{\prime}\right\}=( \pm a) \mathcal{D}^{+} \delta\left(x-x^{\prime}\right) \\
&\left\{I_{-}, H_{+}^{\prime}\right\}=( \pm a) \mathcal{D}^{-} \delta\left(x-x^{\prime}\right)
\end{aligned}
$$

This similar structure, of course, is not just a coincidence but is contingent upon the fact that the models of section 1 and the most general one, obeying our requirements (1), (2) and (3) (resp. (3a)), are related by a simple conformal transformation (cf. ref. [38], section $5.5)$.

For completeness we present the action of the most general "genuine" supergravity model as obtained on the basis of the gPSM formalism. Inserting the corresponding Poisson tensor (5.15)-(5.17) into eq. (1.1) leads to

$$
\begin{aligned}
\mathcal{S}=\int_{\mathcal{M}}\left(\phi \mathrm{d} \omega+X^{a} D e_{a}\right. & +\chi^{\alpha} D \psi_{\alpha}+\epsilon\left(V+Y Z-\frac{1}{2} \chi^{2}\left(\frac{V Z+V^{\prime}}{2 u}+\frac{\tilde{u}_{0}^{2} V^{2}}{2 u^{3}}\right)\right) \\
+ & \frac{Z}{4} X^{a}\left(\chi \gamma_{a} \gamma^{b} e_{b} \gamma^{3} \psi\right)-\frac{i \tilde{u}_{0} V}{2 u}\left(\chi \gamma^{a} e_{a} \psi\right) \\
& \left.-\frac{i \tilde{u}_{0}}{2} X^{a}\left(\psi \gamma_{a} \psi\right)-\frac{1}{2}\left(u+\frac{Z}{8} \chi^{2}\right)\left(\psi \gamma_{3} \psi\right)\right)
\end{aligned}
$$


Starting from a bosonic gravity theory constructed according to the principles outlined in section 1 and equipped with a potential as given in (1.6), by our analysis this is its unique $N=(1,1)$ supergravity extension and it contains the minimal number of fields: The bosonic variables $\left(\omega, e_{a}\right)$ and $\left(\phi, X^{a}\right)$ are supplemented by a gravitino $\psi_{\alpha}=\psi_{\alpha m} \mathrm{~d} x^{m}$ and a dilatino $\chi^{\alpha}$, only. No auxiliary fields appear, nevertheless (6.6) has a (relatively) simple structure.

\section{Conclusions}

The underlying symmetries in the PSM-formulation produce a simple, albeit nonlinear algebra of Hamiltonian constraints which is difficult to analyze from the point of view of supersymmetry and supergravity. However, as noticed some time ago for $2 \mathrm{~d}$ bosonic gravity theories [44] by a linear transformation of the constraints this algebra can be cast into the one appearing for the constraints in the ADM-analysis ("Virasoro type algebra"), supplemented by the Lorentz constraint in a simple manner, when the deformation by the dilaton field becomes relevant. In two dimensions such an algebra in the parlance of the string community is also called a "conformal algebra". Here we have used this method for the graded case.

A natural set of requirements for a "genuine" supergravity theory ("superconformal gravity") implies that rigid supersymmetry appears in some flat limit and that some obvious main features of the superconformal algebra should survive in the deformed one. Starting from the requirement (1) of section 1 (rigid supersymmetry in the flat limit), its implementation together with (2) determines the proper choice for the constraints $H_{( \pm)}$ and $I_{( \pm)}$to be used in the dilaton supergravity algebra. While (1) and (2) do not yet lead to restrictions on the relation between the bosonic potential $v$ and the prepotential $u$, requirement (3) of sect. 11, resp. (3a) of sect. 3, $\left(\left\{I_{(+)}, I_{(+)}^{\prime}\right\}=-2 H_{(+)} \delta\left(x-x^{\prime}\right)\right.$, etc.) leads to the mathematical condition (5.1). Its application drastically reduces the class of allowed Poisson tensors and exhibits very gratifying properties:

- gPSM supergravity leads to a unique fermionic extension of all bosonic PSM theories of gravity, whose potential, however, is forced to be, at most, quadratic in torsion (cf. (6.6) ). Moreover the bosonic potential must be derivable from a prepotential (cf. (5.11)).

- The supergravity algebra of these general supergravities does not show the singularities and obstructions, which in the the generic case of gPSMs are present.

- The most general supergravity action allowed by the requirements does not lead to any new singularities in the fermionic extension.

The bosonic models which possess this unique supergravity extension are precisely the one which include spherically reduced gravity, the (bosonic) stringy Black Hole and other physically motivated theories. It should be emphasized that none of the final "fortious" results regarding uniqueness and the absence of singularities and obstructions were introduced in an ad-hoc manner. 
The present argument has been applied to the $N=(1,1)$ supergravities of refs. [38,40]. Spherical reduction of $N=1$ supergravity in $d=4$ is known to need Dirac Killing spinors. Therefore, a treatment of that case has to await the extension of the gPSM approach to $N=(2,2)$ which should be possible along the the same lines. It would be especially interesting to check also in that case the consequences of the three requirements imposed in our present work.

Other promising directions of research seem to be the investigation of global properties by means of a super pointparticle for the class of "physical" $2 \mathrm{~d}$ supergravity theories determined here and the coupling of matter fields. The superparticle in supergravity so far has been formulated in the superfield formalism only. To this end the relation of gPSM supergravity to superspace supergravity should be clarified. So far, this has been done only partially $[40,51,55]$, namely for models with $Z=0$ in (1.6), related to a non-dynamical dilaton field in the equivalent action (1.11). Recent calculations for non-vanishing bosonic torsion $(Z \neq 0)$ indicate that there exists a close relation between precisely the gPSM supergravities found in this work and the dilaton supergravity formulated in superspace [52]. As the determination of general classical solutions and the quantization in the PSM formulation is a well-established field $[7,48]$, this opens new perspectives for dilaton superfield supergravity [39], problems for which complete solutions involving the fermionic sector do not seem to exist in the literature.

\section{Acknowledgement}

The authors are grateful especially to D. Grumiller for stimulating discussions which led us to consider the approach of this paper. They thank T. Strobl for illuminating comments. The work has been supported by the Swiss National Science Foundation (SNF) and the Austrian Science Foundation (FWF) Project 14650-TPH and P-16030-N08.

\section{A. Notations and conventions}

In this technical Appendix notations and conventions are summarized briefly. They are identical to those in ref. [38,40], where additional explanations can be found.

A rather elaborate labelling of indices is used to distinguish different combinations of objects:

$\mathbf{I}, \mathbf{J}, \mathbf{K}$ Capital Latin letters from the middle of the alphabet represent a generic index, which can include both, commuting and anti-commuting objects. Commutations of objects are defined in the standard way

$$
v^{I} w^{J}=(-1)^{I J} w^{J} v^{I}
$$

where the indices in the exponent take values 0 (commuting object) or 1 (anticommuting object). In the application to gPSM supergravity the generic index $I$ itself splits into the classes

$$
I=(i, \alpha)=(\phi, a, \alpha)
$$

which are explained below. 
$\mathbf{i}, \mathbf{j}, \mathbf{k}$ Lower case Latin letters from the middle of the alphabet represent a generic commuting object.

$\phi$ The index $\phi$ is used to label the dilaton component of the gPSM fields:

$$
X^{\phi}=\phi \quad A_{\phi}=\omega
$$

$\mathbf{a}, \mathbf{b}, \mathbf{c}$ Lower case Latin letters from the beginning of the alphabet are used as bosonic anholonomic indices. At the level of gPSM supergravity they label the two bosonic one-forms representing the zweibein and the corresponding target-space variables, resp.

$\boldsymbol{\alpha}, \boldsymbol{\beta}, \boldsymbol{\gamma}$ Greek letters from the beginning of the alphabet are (anholonomic) anti-commuting indices. They label the spinor components of the gravitino and the dilatino.

$\mathbf{m}, \mathbf{n}, \mathbf{o}$ To label the components of the world-sheet manifold lower case Latin letters from the middle of the alphabet are used.

The summation convention is always $N W \rightarrow S E$, especially for a fermion $\chi: \chi^{2}=$ $\chi^{\alpha} \chi_{\alpha}$. Our conventions are arranged in such a way that almost every bosonic expression is transformed trivially to the graded case when using this summation convention and replacing commuting indices by general ones. This is possible together with exterior derivatives acting from the right, only. Thus the graded Leibniz rule is given by

$$
\mathrm{d}(A B)=A \mathrm{~d} B+(-1)^{B}(\mathrm{~d} A) B .
$$

In terms of anholonomic indices the metric and the symplectic $2 \times 2$ tensor are defined as

$$
\eta_{a b}=\eta^{a b}=\left(\begin{array}{cc}
1 & 0 \\
0 & -1
\end{array}\right) \quad \epsilon_{a b}=-\epsilon^{a b}=\left(\begin{array}{cc}
0 & 1 \\
-1 & 0
\end{array}\right) \quad \epsilon_{\alpha \beta}=\epsilon^{\alpha \beta}=\left(\begin{array}{cc}
0 & 1 \\
-1 & 0
\end{array}\right)
$$

The metric in terms of holonomic indices is obtained by $g_{m n}=e_{n}^{b} e_{m}^{a} \eta_{a b}$ and for the determinant the standard expression $e=\operatorname{det} e_{m}^{a}=\sqrt{-\operatorname{det} g_{m n}}$ is used. The volume form reads $\epsilon=\frac{1}{2} \epsilon^{a b} e_{b} \wedge e_{a}$.

The $\gamma$-matrices are used in a chiral representation:

$$
\gamma_{\alpha}^{0}{ }^{\beta}=\left(\begin{array}{ll}
0 & 1 \\
1 & 0
\end{array}\right) \quad \gamma_{\alpha}^{1}{ }^{\beta}=\left(\begin{array}{cc}
0 & 1 \\
-1 & 0
\end{array}\right) \quad \gamma_{\alpha}^{3}{ }^{\beta}=\left(\gamma^{1} \gamma^{0}\right)_{\alpha}{ }^{\beta}=\left(\begin{array}{cc}
1 & 0 \\
0 & -1
\end{array}\right)
$$

The matrices $\left(\gamma^{a}\right)^{\alpha \beta}=\epsilon^{\alpha \delta} \gamma^{a}{ }_{\delta}^{\beta}$ and $\left(\gamma^{3}\right)^{\alpha \beta}$ are symmetric in $\{\alpha, \beta\}$ The most important relations among the $\gamma$-matrices are:

$$
\gamma^{a} \gamma^{b}=\eta^{a b} \mathbf{1}+\epsilon^{a b} \gamma^{3} \quad \gamma^{a} \gamma^{3}=\gamma^{b} \epsilon_{b}{ }^{a}
$$

Covariant derivatives of anholonomic indices with respect to the geometry include the two-dimensional spin-connection $\omega^{a b}=\omega \epsilon^{a b}$. When acting on lower indices the explicit expressions read $\left(\frac{1}{2} \gamma^{3}\right.$ is the generator of Lorentz transformations in spinor space):

$$
(D e)_{a}=\mathrm{d} e_{a}-\omega \epsilon_{a}^{b} e_{b} \quad(D \psi)_{\alpha}=\mathrm{d} \psi_{\alpha}+\frac{1}{2} \omega \gamma_{\alpha}^{3}{ }^{\beta} \psi_{\beta}
$$


Finally light-cone components are introduced. As we work with spinors in a chiral representation we can use

$$
\chi^{\alpha}=\left(\chi^{+}, \chi^{-}\right), \quad \chi_{\alpha}=\left(\begin{array}{c}
\chi_{+} \\
\chi_{-}
\end{array}\right) .
$$

For Majorana spinors upper and lower chiral components are related by $\chi^{+}=\chi_{-}, \chi^{-}=$ $-\chi_{+}$. Vectors in light-cone coordinates are given by

$$
v^{++}=\frac{i}{\sqrt{2}}\left(v^{0}+v^{1}\right), \quad v^{--}=\frac{-i}{\sqrt{2}}\left(v^{0}-v^{1}\right) .
$$

Correspondingly the derivatives with respect to these components are written compactly as

$$
\partial_{K}=\frac{\partial}{\partial X^{K}}=\left(\partial_{\phi}, \partial_{++}, \partial_{--}, \partial_{+}, \partial_{-}\right) .
$$

The additional factor $i$ in (A.10) permits a direct identification of the light-cone components with the components of the spin-tensor $v^{\alpha \beta}=\frac{i}{\sqrt{2}} v^{c} \gamma_{c}^{\alpha \beta}$. This implies that $\eta_{++\mid--}=1$ and $\epsilon_{--\mid++}=-\epsilon_{++\mid--}=1$. The $\gamma$-matrices in light-cone coordinates become

$$
\left(\gamma^{++}\right)_{\alpha}{ }^{\beta}=\sqrt{2} i\left(\begin{array}{cc}
0 & 1 \\
0 & 0
\end{array}\right), \quad\left(\gamma^{--}\right)_{\alpha}{ }^{\beta}=-\sqrt{2} i\left(\begin{array}{cc}
0 & 0 \\
1 & 0
\end{array}\right) .
$$

To prevent confusion of indices in tensors we separate them by a vertical line where necessary (e.g. $P^{++\mid+}$is the component $a=++, \alpha=+$ of $P^{a \alpha}$ ).

\section{References}

[1] R. H. Dicke Rev. Mod. Phys. 29 (1957) 29.

[2] P. Jordan, Schwerkraft und Weltall : Grundlagen der theoretischen Kosmologie. Vieweg, second ed., 1955.

[3] P. Jordan, The present state of Dirac's cosmological hypothesis, Z. Phys. 157 (1959) 112-121.

[4] M. Fierz, Über die physikalische Deutung der erweiterten Gravitationstheorie P. Jordans, Helv. Phys. Acta 29 (1956) 128.

[5] C. Brans and R. H. Dicke, Mach's principle and a relativistic theory of gravitation, Phys. Rev. 124 (1961) 925-935.

[6] C. G. Callan, Jr., S. B. Giddings, J. A. Harvey, and A. Strominger, Evanescent black holes, Phys. Rev. D45 (1992) 1005-1009, hep-th/9111056.

[7] D. Grumiller, W. Kummer, and D. V. Vassilevich, Dilaton gravity in two dimensions, Phys. Rept. 369 (2002) 327, http://arXiv.org/abs/hep-th/0204253.

[8] D. Z. Freedman, P. van Nieuwenhuizen, and S. Ferrara, Progress toward a theory of supergravity, Phys. Rev. D13 (1976) 3214-3218.

[9] D. Z. Freedman and P. van Nieuwenhuizen, Properties of supergravity theory, Phys. Rev. D14 (1976) 912. 
[10] S. Deser and B. Zumino, Consistent supergravity, Phys. Lett. B62 (1976) 335.

[11] S. Deser and B. Zumino, A complete action for the spinning string, Phys. Lett. B65 (1976) 369-373.

[12] M. B. Green, J. H. Schwarz, and E. Witten, SUPERSTRING THEORY. Cambridge University Press, 1987. Vol. 1: Introduction.

[13] D. Lüst and S. Theisen, Lectures on String Theory, vol. 346 of Lecture notes in physics. Springer Verlag, Berlin, 1989.

[14] J. Polchinski, String theory. Cambridge University Press, 1998. Vol. I and II.

[15] F. Haider and W. Kummer, Quantum functional integration of nonEinsteinian gravity in $d=$ 2, Int. J. Mod. Phys. A9 (1994) 207-220.

[16] W. Kummer, H. Liebl, and D. V. Vassilevich, Exact path integral quantization of generic 2-d dilaton gravity, Nucl. Phys. B493 (1997) 491-502, [http://arXiv.org/abs/gr-qc/9612012].

[17] W. Kummer, H. Liebl, and D. V. Vassilevich, Non-perturbative path integral of 2d dilaton gravity and two-loop effects from scalar matter, Nucl. Phys. B513 (1998) 723-734, http://arXiv.org/abs/hep-th/9707115.

[18] W. Kummer, H. Liebl, and D. V. Vassilevich, Integrating geometry in general 2d dilaton gravity with matter, Nucl. Phys. B544 (1999) 403-431, hep-th/9809168].

[19] M. O. Katanaev, W. Kummer, and H. Liebl, Geometric Interpretation and Classification of Global Solutions in Generalized Dilaton Gravity, Phys. Rev. D53 (1996) 5609-5618, http://arXiv.org/abs/gr-qc/9511009.

[20] M. O. Katanaev, W. Kummer, and H. Liebl, On the completeness of the black hole singularity in 2d dilaton theories, Nucl. Phys. B486 (1997) 353-370, gr-qc/9602040.

[21] R. B. Mann, A. Shiekh, and L. Tarasov, Classical and quantum properties of two-dimensional black holes, Nucl. Phys. B341 (1990) 134-154.

[22] T. Banks and M. O'Loughlin, Two-dimensional quantum gravity in Minkowski space, Nucl. Phys. B362 (1991) 649-664.

[23] J. Gegenberg and G. Kunstatter, Quantum theory of black holes, Phys. Rev. D47 (1993) 4192-4195, gr-qc/9302006.

[24] J. P. S. Lemos and P. M. Sa, The black holes of a general two-dimensional dilaton gravity theory, Phys. Rev. D49 (1994) 2897-2908, arXiv:gr-qc/9311008.

[25] D. Louis-Martinez, J. Gegenberg, and G. Kunstatter, Exact Dirac quantization of all 2-d dilaton gravity theories, Phys. Lett. B321 (1994) 193-198, gr-qc/9309018.

[26] P. Schaller and T. Strobl, Canonical quantization of non-Einsteinian gravity and the problem of time, Class. Quant. Grav. 11 (1994) 331-346, arXiv:hep-th/9211054.

[27] P. Schaller and T. Strobl, Poisson structure induced (topological) field theories, Mod. Phys. Lett. A9 (1994) 3129-3136, http://arXiv.org/abs/hep-th/9405110.

[28] P. Thomi, B. Isaak, and P. Hájiček, Spherically symmetric systems of fields and black holes. 1. Definition and properties of apparent horizon, Phys. Rev. D30 (1984) 1168.

[29] P. Hájiček, Spherically symmetric systems of fields and black holes. 2. Apparent horizon in canonical formalism, Phys. Rev. D30 (1984) 1178. 
[30] H.-J. Schmidt, A new proof of birkhoff's theorem, Grav. Cosmol. 3 (1997) 185, http://arXiv.org/abs/gr-qc/9709071.

[31] H.-J. Schmidt, A two-dimensional representation of four-dimensional gravitational waves, Int. J. Mod. Phys. D7 (1998) 215-224, http://arXiv.org/abs/gr-qc/9712034.

[32] B. M. Barbashov, V. V. Nesterenko, and A. M. Chervyakov, The solitons in some geometrical field theories, Theor. Math. Phys. 40 (1979) 572-581. Teor. Mat. Fiz. 40 (1979) 15-27, J. Phys. A13 (1980) 301-312.

[33] C. Teitelboim, Gravitation and Hamiltonian structure in two space-time dimensions, Phys. Lett. B126 (1983) 41.

[34] C. Teitelboim, The hamiltonian structure of two-dimensional space-time and its relation with the conformal anomaly, in Quantum theory of gravity: essays in honor of the 60th birthday of Bryce S.DeWitt (S. Christensen, ed.), (Bristol), pp. 327-344, Hilger, 1984.

[35] R. Jackiw, Liouville field theory: a two-dimensional model for gravity, in Quantum theory of gravity : essays in honor of the 60th birthday of Bryce S.DeWitt (S. Christensen, ed.), (Bristol), pp. 327-344, Hilger, 1984.

[36] R. Jackiw, Lower dimensional gravity, Nucl. Phys. B252 (1985) 343-356.

[37] P. S. Howe, Super Weyl transformations in two-dimensions, J. Phys. A12 (1979) 393-402.

[38] M. Ertl, W. Kummer, and T. Strobl, General two-dimensional supergravity from Poisson superalgebras, JHEP 01 (2001) 042, arXiv:hep-th/0012219].

[39] Y.-C. Park and A. Strominger, Supersymmetry and positive energy in classical and quantum two-dimensional dilaton gravity, Phys. Rev. D47 (1993) 1569-1575, arXiv:hep-th/9210017.

[40] M. Ertl, Supergravity in two spacetime dimensions. PhD thesis, Technische Universität Wien, 2001. arXiv:hep-th/0102140.

[41] T. Strobl, Target-superspace in 2d dilatonic supergravity, Phys. Lett. B460 (1999) 87-93, arXiv:hep-th/9906230.

[42] R. Grimm, J. Wess, and B. Zumino, Consistency checks on the superspace formulation of supergravity, Phys. Lett. B73 (1978) 415.

[43] H. Grosse, W. Kummer, P. Presnajder, and D. J. Schwarz, Novel symmetry of nonEinsteinian gravity in two-dimensions, J. Math. Phys. 33 (1992) 3892-3900, hep-th/9205071.

[44] M. O. Katanaev, Canonical quantization of the string with dynamical geometry and anomaly free nontrivial string in two- dimensions, Nucl. Phys. B416 (1994) 563-605, http://arXiv.org/abs/hep-th/0101168.

[45] M. O. Katanaev, Effective action for scalar fields in two-dimensional gravity, Annals Phys. 296 (2002) 1-50, http://arXiv.org/abs/gr-qc/0101033.

[46] R. Arnowitt, S. Deser, and C. W. Misner in Gravitation: An Introduction to Current Research (L. Witten, ed.), Wiley, New York, 1962.

[47] J. M. Izquierdo, Free differential algebras and generic 2d dilatonic (super)gravities, Phys. Rev. D59 (1999) 084017, arXiv:hep-th/9807007.

[48] L. Bergamin, D. Grumiller, W. Kummer, and P. van Nieuwenhuizen in preparation. 
[49] V. O. Rivelles, Topological two-dimensional dilaton supergravity, Phys. Lett. B321 (1994) 189-192, http://arXiv.org/abs/hep-th/9301029.

[50] M. M. Leite and V. O. Rivelles, Topological dilatonic supergravity theories, Class. Quant. Grav. 12 (1995) 627-636, http://arXiv.org/abs/hep-th/9410003.

[51] N. Ikeda, Gauge theory based on nonlinear Lie superalgebras and structure of 2-d dilaton supergravity, Int. J. Mod. Phys. A9 (1994) 1137-1152.

[52] L. Bergamin and W. Kummer in preparation.

[53] M. O. Katanaev and I. V. Volovich, String model with dynamical geometry and torsion, Phys. Lett. B175 (1986) 413-416.

[54] M. O. Katanaev and I. V. Volovich, Two-dimensional gravity with dynamical torsion and strings, Ann. Phys. 197 (1990) 1.

[55] N. Ikeda, Two-dimensional gravity and nonlinear gauge theory, Ann. Phys. 235 (1994) 435-464, arXiv:hep-th/9312059. 